\title{
Reporting and methodological quality of meta-analyses in urological literature
}

\author{
Leilei Xia ${ }^{\text {Corresp., }}{ }^{1}$, Jing Xu ${ }^{2}$, Thomas J Guzzo ${ }^{\text {Corresp. } 1}$ \\ 1 Division of Urology, Department of Surgery, University of Pennsylvania Perelman School of Medicine, Philadelphia, Pennsylvania, United States \\ 2 Cerebral Vascular Disease Research Laboratories, Department of Neurology and Neuroscience Program, University of Miami Miller School of Medicine, \\ Miami, Florida, United States \\ Corresponding Authors: Leilei Xia, Thomas J Guzzo \\ Email address: leilei.xia@uphs.upenn.edu, thomas.guzzo@uphs.upenn.edu
}

Purpose: To assess the overall quality of published urological meta-analyses and identify predictive factors for high quality. Materials and Methods: We systematically searched PubMed to identify meta-analyses published from January $1^{\text {st }}, 2011$ to December $31^{\text {th }}, 2015$ in 10 predetermined major paper-based urology journals. The characteristics of the included meta-analyses were collected, and their reporting and methodological qualities were assessed by the PRISMA checklist (27 items) and AMSTAR tool (11 items), respectively. Descriptive statistics were used for individual items as a measure of overall compliance, and PRISMA and AMSTAR scores were calculated as the sum of adequately reported domains. Logistic regression was used to identify predictive factors for high qualities. Results: A total of 183 meta-analyses were included. The mean PRISMA and AMSTAR scores were $22.74 \pm 2.04$ and $7.57 \pm 1.41$, respectively. PRISMA item 5, protocol and registration, items 15 and 22, risk of bias across studies, items 16 and 23, additional analysis had less than $50 \%$ adherence. AMSTAR item 1, "a priori" design, item 5 , list of studies and item 10, publication bias had less than $50 \%$ adherence. Logistic regression analyses showed that funding support and "a priori" design were associated with superior reporting quality, following PRISMA guideline and "a priori" design were associated with superior methodological quality. Conclusions: Reporting and methodological qualities of recently published meta-analyses in major paper-based urology journals are generally good. Further improvement could potentially be achieved by strictly adhering to PRISMA guideline and having "a priori" protocol. 
1 [Title Page]

2

3 Reporting and methodological quality of meta-analyses in 4 urological literature

6 Authors

7 Leilei Xia ${ }^{1}$, Jing $\mathrm{Xu}^{2}$, Thomas J. Guzzo ${ }^{1}$

8

9 Affiliations

${ }^{1}$ Division of Urology, Department of Surgery, University of Pennsylvania Perelman School of

11 Medicine, Philadelphia, Pennsylvania, U.S.A

$12{ }^{2}$ Cerebral Vascular Disease Research Laboratories, Department of Neurology and Neuroscience

13 Program, University of Miami Miller School of Medicine, Miami, Florida, U.S.A

\section{Correspondence information}

Thomas J. Guzzo, MD, MPH

Perelman Center for Advanced Medicine, West Pavilion, 3rd Floor, 3400 Civic Center Boulevard,

Philadelphia, Pennsylvania 19104

E-mail: thomas.guzzo@uphs.upenn.edu 


\section{ABSTRACT}

23

24

Purpose: To assess the overall quality of published urological meta-analyses and identify predictive factors for high quality.

Materials and Methods: We systematically searched PubMed to identify meta-analyses published from January $1^{\text {st }}, 2011$ to December $31^{\text {th }}, 2015$ in 10 predetermined major paper-based urology journals. The characteristics of the included meta-analyses were collected, and their reporting and methodological qualities were assessed by the PRISMA checklist (27 items) and AMSTAR tool (11 items), respectively. Descriptive statistics were used for individual items as a measure of overall compliance, and PRISMA and AMSTAR scores were calculated as the sum of adequately reported domains. Logistic regression was used to identify predictive factors for high qualities.

Results: A total of 183 meta-analyses were included. The mean PRISMA and AMSTAR scores were $22.74 \pm 2.04$ and $7.57 \pm 1.41$, respectively. PRISMA item 5, protocol and registration, items 15 and 22, risk of bias across studies, items 16 and 23, additional analysis had less than $50 \%$ adherence. AMSTAR item 1, "a priori" design, item 5, list of studies and item 10, publication bias had less than $50 \%$ adherence. Logistic regression analyses showed that funding support and " $a$ priori" design were associated with superior reporting quality, following PRISMA guideline and "a priori" design were associated with superior methodological quality.

Conclusions: Reporting and methodological qualities of recently published meta-analyses in major paper-based urology journals are generally good. Further improvement could potentially be achieved by strictly adhering to PRISMA guideline and having "a priori" protocol. 
50

\section{INTRODUCTION}

A systematic review is a review of a clearly formulated question using systematic methods to identify, select and critically appraise relevant research. The systematic review may include a quantitative synthesis of results called meta-analysis, which summarizes all results of primary studies in order to obtain a combined estimate of the effect. Certain types of systematic review and meta-analysis are considered as the highest level of evidence (level 1a) (http://www.cebm.net/oxford-centre-evidence-based-medicine-levels-evidence-march-2009/).

Also, well-conducted meta-analyses can sometimes resolve conflicting evidence and provide more reliable conclusions (Berlin \& Golub, 2014). Meta-analyses are often appealing to both authors and journals as they are commonly highly cited publication. Rapidly expanding literature across all the medical disciplines raise the increasing need to summarize and synthesis the currently available evidence. Such factors have contributed to the increased number of published metaanalyses in medical journals (Tunis et al., 2013; Zhang et al., 2016).

However, like original research articles, quantity does not mean quality (Adie et al., 2015; Berlin \& Golub, 2014; Dechartres et al., 2014; Murad \& Montori, 2013). It is imperative for both the medical and publishing community to aware the negative influence of flawed or low-quality metaanalyses (Berlin \& Golub, 2014). Several statements or guidelines have been proposed and validated as the tools to assess the quality of published meta-analyses (Faggion, 2015; Liberati et al., 2009; Moher et al., 2009; Pieper et al., 2015; Shea et al., 2007a; Shea et al., 2007b; Shea et al., 2009; Stroup et al., 2000). The most well-known guideline is the Preferred Reporting Items for Systematic Reviews and Meta-Analyses (PRISMA), which is actually a checklist recommended to follow when reporting meta-analyses (Liberati et al., 2009; Moher et al., 2009). An earlier initiative was the development of the AMSTAR, a measuring tool to assess the methodological or conducting quality of meta-analyses (Shea et al., 2007b; Shea et al., 2009). In other words, AMSTAR usually serves as a critical appraisal tool to identify the scope of bias in methodology at the review level.

Although still debatable, a number of studies in various surgical and medical fields have used the "scores" based on the fulfillments of PRISMA and AMSTA to assess the qualities of systematic 
80

81

82

83

84

85

86

87

88

89

90

91

92

93

94

95

96

97

98

99

100

101

102

103

104

105

106

107

108

109

110

reviews and meta-analyses (Adie et al., 2015; Gagnier \& Kellam, 2013; Liu et al., 2017; Shea et al., 2007a; Tunis et al., 2013; Zhang et al., 2016). There exist several duplicate items in the two tools, generally they are considered separate tools and are commonly used together for the assessment, PRISMA for reporting quality and AMSTAR for methodological quality (Adie et al., 2015; Gagnier \& Kellam, 2013; Liu et al., 2017; Shea et al., 2007a; Tunis et al., 2013; Zhang et al., 2016).

To date, no studies have comprehensively assessed the reporting and methodological quality of urological meta-analyses, in particular those published after the PRISMA initiative (2009). Considering meta-analyses are often influential and highly cited publications, there is a need to explore whether general characteristics (author, journal, and report) of the meta-analyses have an association with the overall quality. Therefore, in the present study, we specifically focused on meta-analyses published in major paper-based urology journals, with the aim to assess the reporting and methodological quality, as well as to identify relevant predictive or associated factors.

\section{METHODS}

\section{Eligibility criteria}

To be eligible for inclusion, a meta-analysis had to meet the following inclusion criteria: (1) a study with the meta-analytic methodology pooling results from primary articles (including metaanalysis alone or systematic reviews containing meta-analyses); (2) published in the following 10 predetermined urology journals: British Journal of Urology International (BJUI), European Urology (EU), Journal of Endourology (JEU), Journal of Pediatric Urology (JPU), Journal of Urology (JU), Neurourology and Urodynamics (NUUD), Urology (URO), Urolithiasis (UL), formerly known as Urological Research, Urologic Oncology (UO) and World Journal of Urology (WJU); (3) published in the printed journal between January $1^{\text {st }} 2011$ to December 31th 2015 (excluding "Epub ahead of print").

Exclusion criteria were: (1) systematic review without meta-analysis; (2) original research article or original research article combined with a meta-analysis; (3) network meta-analysis or multiple 
111 group comparison meta-analysis; (4) meta-analysis of single proportions; (5) meta-analysis

112 originally published in the Cochrane Database of Systematic Reviews. There were two reasons for

113 our decision to exclude the network meta-analysis (multiple group comparison meta-analysis) and

114 meta-analysis of single proportions. First, they were relatively uncommon compared to other

115 "traditional" meta-analyses. Second, the methods and results reported by those meta-analyses were

116 very heterogenic and different from "traditional" or pairwise meta-analyses (Bafeta et al., 2013;

117 Bafeta et al., 2014). Some of the items in the PRISMA and AMSTAR do not perfectly apply to 118 network meta-analyses and meta-analysis of single proportions.

119

120

121

122

123

124

125

126

127

128

129

130

131

132

133

134

135

136

137

138

139

140

Two investigators (LX, JX) independently screened the titles and abstracts of all the identified references. Full-text were then retrieved for potential eligible meta-analysis. Discrepancies were resolved by discussion between the two investigators.

\section{Search strategy}

The objective was to identify all the meta-analyses published from January $1^{\text {st }}, 2011$ to December $31^{\text {th }}, 2015$ in 10 predetermined major paper-based urology journals: BJUI, EU, JEU, JPU, JU, NUUD, URO, UL, UO, and WJU. We performed a focused search on PubMed. For BJUI, the search strategy was: ("BJU international"[Journal]) AND (meta-analysis [Title/Abstract] OR systematic review [Title/Abstract]) AND ("2011/01/01"[Date - Publication]: "2015/12/31"[Date - Publication]). For other journals, the search strategy is the same except for the journal name.

\section{Data extraction}

We collected all data on general characteristics of the included meta-analyses, and the key reporting (PRISMA) and methodological (AMSTAR) components of the meta-analysis process. Two investigators (LX, JX) independently extracted the data. Any disagreements were resolved by discussion between the two investigators. Inter-observer reliability was examined using the kappa ( $\kappa)$ value. 
142

143

144

145

146

147

\section{General characteristics}

We collected data on the following general characteristics: (1) corresponding author's region and country; (2) number of authors; (3) presence or absence of a professional with the background of epidemiology or statistics as a coauthor (including the acknowledgement part); (4) number of participating centers (department level); (5) subspecialties in urology (based on the American Urological Association classification); (6) presence or absence of any funding source; (7) the number of included studies; (8) type of the included studies (only RCTs or RCTs plus non-RCTs or only non-RCTs); (9) type of the meta-analyses (interventional, diagnostic, incidence related, prognostic or cannot classify); (10) type of the interventional meta-analyses (surgical or nonsurgical); (11) attached a PRISMA checklist or not; (12) followed the PRISMA guideline or not (claimed this in the article or not); (13) provided the protocol and registration information or not, which also referred to the PRISMA item 5; (14) "a priori" design or not (claimed this in the article or not), which also referred to the AMSTAR item 1.

\section{Assessment of key reporting components in the meta-analysis process}

The PRISMA statement is a checklist of 27 items that are recommended to be included in systematic review and meta-analysis to ensure that the published report contains all relevant information (Supplemental Information). The present study focused only on meta-analyses and every item was applicable. Each PRISMA item was rated with a "yes" or "no" response. A "yes" response means that the item was reported, and a "no" response means that the item was not reported. For the purpose of data analysis, reported points were assigned as follows: "yes" = 1, "no" $=0$. Therefore every included meta-analysis had an overall PRISMA score rated out of a maximum score of 27.

\section{Assessment of key methodological components in the meta-analysis process}

The AMSTAR tool is an 11-item questionnaire that was used to determine the methodological or conducting quality of systematic reviews and meta-analyses (Supplemental Information). The original tool had four responses with each item, "yes", “no", “can't answer', or "'not 
172 applicable', Due to the fact that we focused on meta-analyses (excluded pure systematic reviews), 173 every item was applicable. Each AMSTAR item was rated with a "yes" "no" or "cannot

174

175

176

177

178

179

180

181

182

183

184

185

186

187

188

189

190

191

192

193

194

195

196

197

198

199

200

201 answer" response. A "yes" response means that the item is fulfilled, a "no" response means that the item is not fulfilled, a "can't answer" response means that it is inconclusive as to whether the item is fulfilled. For the purpose of data analysis, reported points were assigned as follows: "yes" $=1$, "no" or "can't answer" $=0$. Therefore every included meta-analysis had an overall AMSTAR score rated out of a maximum score of 11 .

\section{Data analysis}

Analyses, tables, and figures were configured by using a spreadsheet program (Excel 2013, Microsoft) and a statistical software (STATA 14.0, StataCorp LP). A descriptive analysis was performed for PRISMA and AMSTAR scores grouped by multiple categories. Shapiro-Wilk test was used to assess the normality of the PRISMA and AMSTAR scores ( $p=0.376$ and $p=0.057$, respectively). Based on the distributions of PRISMA and AMSTAR scores and Shapiro-Wilk test results, we used the parametric tests to compare the qualities. Comparisons of mean qualities between dichotomous factors were conducted using the independent Student's t-test. Comparisons of study qualities between multifactor variables were conducted using the one-way analysis of variance (ANOVA), with the Tukey's HSD post hoc test. The PRISMA score and AMSTAR score were both divided into the superior and inferior quality groups with a cutoff value of $75 \%$ percentile of the respective ranges. Univariate logistic regression analysis was used to compare the differences between the superior and inferior groups with potential factors affecting study qualities. Variables included continent origin, country origin, number of authors, presence or absence of a professional with the background of epidemiology or statistics as a coauthor, number of participating centers, subspecialties, funding support, number of included studies, type of the included studies, interventional meta-analysis, type of interventional meta-analysis, followed the PRISMA guideline, and "a priori" design. Factors that found to be significant $(p<0.1)$ were then entered into the multivariate logistic regression analysis. A p $<0.05$ was considered significant on statistical analyses. All the analyses were two-sided. 
202

203

204

205

206

207

208

209

210

211

212

213

214

215

216

217

218

219

220

221

222

223

224

225

226

227

228

229

230

231

232

\section{RESULTS}

\section{Search results}

Figure 1 depicts a flow diagram of meta-analyses selection. The initial search identified 641 references with potential relevance. Screening the title and abstract excluded 422 references and another 36 references were excluded after reviewing the full-text. Finally, 183 meta-analyses were included for the final assessment and analysis (Supplemental Information).

\section{General characteristics of the meta-analyses}

The characteristics of the 183 meta-analyses are shown in Table 1. The number of authors and number of included studies were divided into two groups with the cutoff setting at median values ( 7 and 10, respectively). The number of patients included per meta-analysis ranged from 152 to 4082606. The number of patients in 14 studies could not be determined. EU ( $\mathrm{n}=44,24 \%)$ published the largest number of included meta-analyses and JPU ( $n=2,1 \%)$ had the lowest number. The region where the largest number of included meta-analyses originated was Asia $(\mathrm{n}=$ $89,49 \%)$. The most common countries of publication were China $(n=82,45 \%)$, the USA $(n=22$, $12 \%)$, and the UK $(\mathrm{n}=20,11 \%)$. Forty-four $(24 \%)$ meta-analyses had at least one professional with the background of epidemiology or statistics as the coauthor (including the acknowledgement part). The most common subspecialty of the included meta-analyses was urologic oncology $(\mathrm{n}=$ $75,41 \%)$. Most of the included meta-analyses could be categorized as interventional ( $\mathrm{n}=141$, $77 \%)$, which were further subcategorized as surgical intervention (96/141, 68\%) and non-surgical intervention (45/141, 32\%). Sixty-two (34\%) meta-analyses included only RCTs, 71 (39\%) included only non-RCTs, and another 50 (27\%) included both RCTs and non-RCTs. Fifty-five (30\%) meta-analyses received funding support. Only $2(1 \%)$ meta-analyses attached the PRISMA checklist. Fifty-five (30\%) meta-analyses claimed followed the PRISMA guideline. Eight (4\%) meta-analyses provided the protocol and registration information (PRISMA item 5) and 22 metaanalyses claimed the "a priori" design (AMSTAR item 1).

\section{Reporting quality (PRISMA)}


233 The overall mean PRISMA score of all the included meta-analyses was $22.74 \pm 2.04(84.2 \%$ of

234 items adequately reported, on average). Most of the PRISMA items (25 out of 27) had a $\kappa$ value 235 more than 0.65 and none of them had a $\kappa$ value less than 0.5 . Table 1 shows mean PRISMA scores 236 grouped with various factors. After excluding journals (JPU, NUUD, and UO) with less than 10 237 included meta-analyses, EU had the highest mean PRISMA score (23.52 \pm 1.93$)$. However, one238 way ANOVA of PRISMA score showed no significant difference between the 7 journals (BJUI, 239 EU, JEU, JU, URO, WJU, and UL), F $(6,165)=1.71, p=0.12$. Student's t-test showed no 240 significant difference of PRISMA score between the meta-analyses from Asia and those from non241 Asia region, $\mathrm{t}(181)=-0.50, \mathrm{p}=0.62$. There was no significant difference in PRISMA scores 242 between the meta-analyses from China and remaining countries, $\mathrm{t}(181)=-0.15, \mathrm{p}=0.88$. Included 243 meta-analyses in the subspecialty of urologic oncology had higher PRISMA scores than other 244 specialties, $\mathrm{t}(181)=-2.09, \mathrm{p}=0.037$. There was no significant difference in PRISMA scores 245 between the included studies type (Only RCT vs. RCT \& non-RCT vs. Only non-RCT), F (2, 180) $246=0.47, \mathrm{p}=0.63$. There was no significant difference in PRISMA scores between the interventional 247 and non-interventional meta-analyses, $\mathrm{t}(181)=1.80, \mathrm{p}=0.07$. All other two-group comparison 248 test results are shown in Table 1.

Figure 2a shows the PRISMA results on a per-item basis. Per-item PRISMA analysis revealed that 5 items had less than 50\% adherence out of the 183 included meta-analyses (item 5, protocol and registration; items 15 and 22, risk of bias across studies; items 16 and 23, additional analysis). Item 8 (search) also had only $51 \%$ adherence.

254

\section{Methodological quality (AMSTAR)}

256

257 The overall mean AMSTAR score of all the included meta-analyses was $7.57 \pm 1.41(68.8 \%$ of items adequately reported, on average). Most of the AMSTAR items (10 out of 11) had a $\kappa$ value more than 0.65 and none of them had a $\kappa$ value less than 0.5 . Table 1 shows mean AMSTAR scores grouped with various factors. After excluding journals (JPU, NUUD, and UO) with less than 10 included meta-analyses, EU had the highest mean AMSTAR score (7.98 \pm 1.47$)$. One-way ANOVA of AMSTAR score showed significant differences between the 7 journals (BJUI, EU, JEU, JU, URO, WJU, and UL), F $(6,165)=3.03, \mathrm{p}=0.008$. Tukey's HSD post hoc test only 
264 showed that EU had higher AMSTAR score than URO, p =0.034. Student's t-test showed no 265 significant difference in AMSTAR score between the meta-analyses from Asia and those from

266

267

268

269

270

271

272

273

274

275

276

277

278

279

280

281

282

283

284

285

286

287

288

289

290

291

292

293

294

non-Asia region, $\mathrm{t}(181)=0.06, \mathrm{p}=0.95$. There was no significant difference of AMSTAR score between the meta-analyses from China and those from remaining countries, $\mathrm{t}(181)=-0.25, \mathrm{p}=$ 0.80. Unlike PRISMA score, the AMSTAR score of included meta-analyses in the subspecialty of urologic oncology did not differ from other subspecialties, $\mathrm{t}(181)=-0.68, \mathrm{p}=0.50$. There was a significant difference of AMSTAR score between the included studies type (Only RCT vs. RCT $\&$ non-RCT vs. Only non-RCT), F $(2,180)=4.98, \mathrm{p}=0.008$. Tukey's HSD post hoc test showed that meta-analyses included only RCTs had higher AMSTAR score than those included only nonRCTs, $p=0.002$. There was no significant difference of AMSTAR score between the interventional and non-interventional meta-analyses, $\mathrm{t}(181)=-1.23, \mathrm{p}=0.22$. All the other two-group comparison test results are shown in Table 1.

Figure $2 \mathrm{~b}$ shows the AMSTAR results on a per-item basis. Per-item AMSTAR analysis revealed that 3 items had less than 50\% adherence out of the 183 included meta-analyses (item 1, "a priori" design; item 5, list of studies; item 10, publication bias). Item 4 (gray literature) also had only 51\% adherence.

\section{Univariate and multivariate analyses}

The 75\% percentile of the respective ranges of PRISMA score and AMSTAR score was 24 and 9, respectively. The reporting quality (PRISMA) and methodological quality (AMSTAR) were divided by the cutoff value of $75 \%$ percentile into superior quality and inferior quality. The results of univariate and multivariate logistic regression analyses on the PRISMA and AMSTAR scores are presented in Tables 2 and 3, respectively.

Univariate regression analyses demonstrated the following factors to be associated with superior reporting quality (PRISMA score $\geq 24$ ) of the published meta-analyses: subspecialty of urologic oncology, funding support, non-interventional meta-analyses, following PRISMA guideline, and "a priori" design. Multivariate regression analyses confirmed the following factors to be 
295

296

297

298

299

300

301

302

303

304

305

306

307

308

309

310

311

312

313

314

315

316

317

318

319

320

321

322

323

324

325

associated with superior reporting quality (PRISMA score $\geq 24$ ) of the published meta-analyses: funding support and "a priori" design.

Univariate regression analyses demonstrated the following factors to be associated with superior methodological quality (AMSTAR score $\geq 9$ ) of the published meta-analyses: number of authors, subspecialty of urologic oncology, following PRISMA guideline, and "a priori" design. Multivariate regression analyses confirmed the following factors to be associated with superior methodological quality (AMSTAR score $\geq 9$ ) of the published meta-analyses: following PRISMA guideline, and "a priori" design.

\section{DISCUSSION}

Our study demonstrates that both reporting and methodological qualities of recently published meta-analyses in major urology journals were generally good. Also, there were no significant variations of the qualities between major urology journals. On average, PRISMA score was 22.74 $(84.2 \%)$ out of 27 and AMSTAR score was 7.57 (68.8\%) out of 11 . However, there still may be room for improvement based on the per-item results (Figure 2). More importantly, several potential predictive factors for superior quality of urological meta-analyses were identified, including funding support, following PRISMA guideline, and "a priori" design. Knowledge and identification of variables predictive of high-quality meta-analysis are not only useful to readers, but also would be useful for journal reviewers and editors.

Reporting and methodological qualities of meta-analyses in other medical disciplines were evaluated with similar methods (Liu et al., 2017; Zhang et al., 2016). Zhang et al. (Zhang et al., 2016) focused on meta-analyses of surgical interventions in year 2013 and showed the mean PRISMA and AMSTAR adherences (by items) were 22.3 and 7.9, respectively. A recent study showed the mean PRISMA and AMSTAR adherences (by items) in the leading gastroenterology and hepatology journals were 20.8 and 7.6, respectively (Liu et al., 2017). Generally speaking, the quality of meta-analyses in major urology journals are good and consistent with previous studies in other medical fields. 
326 Strengths of our study include the focused search and selection of meta-analyses, comprehensive

327 assessment, and planned logistic regression analyses. We only included meta-analyses because

328 they are different from qualitative systematic reviews in several ways and often have a more

329 consistent format. In addition, some of the assessment items only applied to meta-analyses, such

330 as PRISMA items 14, 15, 16, 21, 22, 23, and AMSTAR items 9 and 10. By excluding systematic

331 reviews without meta-analyses, our results would have more credibility and our conclusions would

332 have a more specific implication. Also, we only focused on meta-analyses published starting from

333 2011, which is one year and a half after the publication of PRISMA statement (July 2009) (Moher

334 et al., 2009). Since the AMSTAR tool was first published in 2007, this timeline setting would 335 possibly minimize the confounding from authors' unavailability of PRISMA checklist and 336 AMSTAR tool themselves.

337

338

There are some limitations to our study. First, the cumulative scores calculated from PRISMA checklist and AMSTAR tool may not be valid or truly reflect the reporting and methodological quality. However, at least for now, the scoring method seems to be the best option to quantify the quality of and meta-analyses (Adie et al., 2015; Gagnier \& Kellam, 2013; Tunis et al., 2013; Zhang et al., 2016). Second, we limited our search to 10 predetermined major paper-based urology journals, which could cause the omitting of some urological meta-analyses published in general medical journals. Our selection of journals was somewhat arbitrary, even though we took the equal distribution of general urology journals (BJUI, EU, JU, URO, and WJU) and subspecialty journals (JEU, JPU, NUUD, UL, and UO) into consideration.

Reporting and methodical weakness of urological meta-analyses were identified through per-item analyses. PRISMA item 15, 22 and AMSTAR item 10 is about publication bias, one of the most important reporting biases in meta-analyses (Sedgwick, 2015). Ideally, for meta-analyses with more than 10 included studies, funnel plots and tests for funnel plot asymmetry should be provided to explore the publication biases (Sterne et al., 2011). PRISMA items 16 and 23 (additional analyses) represent another important area for improvement. Less than 50\% percent meta-analyses conducted additional analysis, such as subgroup analysis, meta-regression, and sensitivity analysis. Meta-analyses often have the intrinsic limitation of heterogeneity and conclusions could be 
$357 \quad I^{2}$ value or other tests, the source of heterogeneity was not routinely explored. Subgroup analysis 358 and meta-regression can be performed to explain the source of the significant heterogeneity (Phan 359 et al., 2015; Thompson, 1994). In addition, sensitivity analyses is the method of choice to explore 360 the robustness of the meta-analysis results. PRISMA item 8 (search) and AMSTAR item 5 (list of 361 studies) were also underreported possibly because of the authors' unawareness. Underreported 362 meta-analyses gave partial search strategies such as keywords used or MeSH terms. However, 363 failing to provide detailed or exact search strategy makes it difficult to repeat the search process. 364 Similarly, AMSTAR item 5 (list of studies) were underreported because authors only considered the lists of included studies and neglected the lists of important excluded studies. Suboptimal compliance with AMSTAR item 4 (gray literature) should also be noted since exclusion of gray literature from meta-analyses can lead to exaggerated estimates of intervention effectiveness (McAuley et al., 2000). In theory, meta-analyses should attempt to identify and retrieve all potentially eligible studies, including gray literature and foreign language literature (McAuley et al., 2000; Phan et al., 2015). In reality, however, considerable additional resources are required, which makes it difficult to finish the process.

372

Multivariate logistic regression showed that claiming a meta-analysis followed the PRISMA statement in the article could potentially predict higher methodological quality (AMSTAR). Although PRISMA guideline is intended to define how to report meta-analyses, it can also be used to design meta-analyses in some way. One worrisome fact is that only 55 of the 183 included metaanalyses claimed followed the PRISMA statement (Table 1). Although it is possible that some of the authors may not have described it in the meta-analysis even if they referred to the PRISMA statement, it is also likely that many authors lacked the knowledge of the availability of PRISMA checklist. Two other facts are that only one journal (EU) endorses the compliance with the PRISMA statement in the instructions for authors and none of the journals required a PRISMA checklist for the initial manuscript submission. Therefore requiring a mandatory PRISMA checklist during submission might improve reporting and methodological quality of metaanalyses. It might be easier to initiate the process from the journals than the authors since only 2 of the 183 included meta-analyses attached the PRISMA checklist (Tewari et al., 2012; van Die 386 et al., 2014). In addition, having a PRISMA checklist makes the peer review process more efficient 387 and more informed. 
Another important predictor of high-quality meta-analyses is "a priori" design. As one of the

390

391

392

393

394

395

396

397

398

399

400

401

402

403

404

405

406

407

408

409

410

411

412

413

414

415

416

417

418

419

420

421

422

423
AMSTAR items, "a priori" design can predict both the reporting quality and methodological quality. In PRISMA checklist, item 5 is "protocol and registration", which can be considered as the higher standard of "a priori" design. It is not hard to understand that "a priori" design can make sure the researchers have a clear thinking and well-organized action. In addition, having a protocol or "a priori" design can partially obligate the authors from post hoc modification of inclusion criteria and analytic methods (Tunis et al., 2013). However, only 8 meta-analyses fulfilled the PRISMA item 5 and only 22 meta-analyses claimed "a priori" design. In the medical publication, requiring the protocol and registration information for RCTs is very common. As for systematic reviews, only Cochrane reviews require the authors to publish a peer-reviewed protocol before conducting the review. Previous studies have shown that Cochrane reviews appear to have higher methodological quality than systematic reviews or meta-analyses published in paper-based journals. Another common registration platform for systematic reviews and meta-analyses is PROSPERO (Booth et al., 2012). It would be very difficult for paper-based journals ask for prospective registration or peer-reviewed protocol for every meta-analysis. Attaching a study protocol written "a priori" might be a good start (Reeves et al., 2015).

\section{CONCLUSIONS}

Reporting and methodological qualities of recently published meta-analyses in major urology journals are generally good, however there are areas for potential improvement. Further improvement could potentially be achieved by strictly adhering to PRISMA guideline and preparing "a priori" protocol.

\section{REFERENCES}

Adie S, Ma D, Harris IA, Naylor JM, and Craig JC. 2015. Quality of conduct and reporting of meta-analyses of surgical interventions. Ann Surg 261(4):685-694. 10.1097/SLA.0000000000000836

Bafeta A, Trinquart L, Seror R, and Ravaud P. 2013. Analysis of the systematic reviews process in reports of network meta-analyses: methodological systematic review. BMJ 347(f3675. 10.1136/bmj.f3675

Bafeta A, Trinquart L, Seror R, and Ravaud P. 2014. Reporting of results from network meta-analyses: methodological systematic review. BMJ 348(g1741. 10.1136/bmj.g1741

Berlin JA, and Golub RM. 2014. Meta-analysis as evidence: building a better pyramid. JAMA 312(6):603-605. 10.1001/jama.2014.8167

Booth A, Clarke M, Dooley G, Ghersi D, Moher D, Petticrew M, and Stewart L. 2012. The nuts and bolts of PROSPERO: 
an international prospective register of systematic reviews. Syst Rev 1(2. 10.1186/2046-4053-1-2

Dechartres A, Altman DG, Trinquart L, Boutron I, and Ravaud P. 2014. Association between analytic strategy and estimates of treatment outcomes in meta-analyses. JAMA 312(6):623-630. 10.1001/jama.2014.8166

Faggion CM, Jr. 2015. Critical appraisal of AMSTAR: challenges, limitations, and potential solutions from the perspective of an assessor. BMC Med Res Methodol 15(63. 10.1186/s12874-015-0062-6

Gagnier JJ, and Kellam PJ. 2013. Reporting and methodological quality of systematic reviews in the orthopaedic literature. J Bone Joint Surg Am 95(11):e771-777. 10.2106/JBJS.L.00597

Liberati A, Altman DG, Tetzlaff J, Mulrow C, Gotzsche PC, loannidis JP, Clarke M, Devereaux PJ, Kleijnen J, and Moher D. 2009. The PRISMA statement for reporting systematic reviews and meta-analyses of studies that evaluate health care interventions: explanation and elaboration. Ann Intern Med 151(4):W65-94.

Liu P, Qiu Y, Qian Y, Chen X, Wang Y, Cui J, and Zhai X. 2017. Quality of meta-analyses in major leading gastroenterology and hepatology journals: A systematic review. J Gastroenterol Hepatol 32(1):39-44. 10.1111/jgh.13591

McAuley L, Pham B, Tugwell P, and Moher D. 2000. Does the inclusion of grey literature influence estimates of intervention effectiveness reported in meta-analyses? Lancet 356(9237):1228-1231. 10.1016/S01406736(00)02786-0

Moher D, Liberati A, Tetzlaff J, Altman DG, and Group P. 2009. Preferred reporting items for systematic reviews and meta-analyses: the PRISMA statement. PLoS Med 6(7):e1000097. 10.1371/journal.pmed.1000097

Murad MH, and Montori VM. 2013. Synthesizing evidence: shifting the focus from individual studies to the body of evidence. JAMA 309(21):2217-2218. 10.1001/jama.2013.5616

Phan K, Tian DH, Cao C, Black D, and Yan TD. 2015. Systematic review and meta-analysis: techniques and guide for the academic surgeon. Ann Cardiothorac Surg 4(2):112-122. 10.3978/j.issn.2225-319X.2015.02.04

Pieper D, Buechter RB, Li L, Prediger B, and Eikermann M. 2015. Systematic review found AMSTAR, but not R(evised)AMSTAR, to have good measurement properties. J Clin Epidemiol 68(5):574-583. 10.1016/j.jclinepi.2014.12.009

Reeves F, Preece P, Kapoor J, Everaerts W, Murphy DG, Corcoran NM, and Costello AJ. 2015. Preservation of the neurovascular bundles is associated with improved time to continence after radical prostatectomy but not long-term continence rates: results of a systematic review and meta-analysis. Eur Urol 68(4):692-704. 10.1016/j.eururo.2014.10.020

Sedgwick P. 2015. What is publication bias in a meta-analysis? BMJ 351(h4419. 10.1136/bmj.h4419

Shea BJ, Bouter LM, Peterson J, Boers M, Andersson N, Ortiz Z, Ramsay T, Bai A, Shukla VK, and Grimshaw JM. 2007 a. External validation of a measurement tool to assess systematic reviews (AMSTAR). PLoS One 2(12):e1350. 10.1371/journal.pone.0001350

Shea BJ, Grimshaw JM, Wells GA, Boers M, Andersson N, Hamel C, Porter AC, Tugwell P, Moher D, and Bouter LM. 2007b. Development of AMSTAR: a measurement tool to assess the methodological quality of systematic reviews. BMC Med Res Methodol 7(10. 10.1186/1471-2288-7-10

Shea BJ, Hamel C, Wells GA, Bouter LM, Kristjansson E, Grimshaw J, Henry DA, and Boers M. 2009. AMSTAR is a reliable and valid measurement tool to assess the methodological quality of systematic reviews. $J$ Clin Epidemiol 62(10):1013-1020. 10.1016/j.jclinepi.2008.10.009

Sterne JA, Sutton AJ, loannidis JP, Terrin N, Jones DR, Lau J, Carpenter J, Rucker G, Harbord RM, Schmid CH, Tetzlaff J, Deeks JJ, Peters J, Macaskill P, Schwarzer G, Duval S, Altman DG, Moher D, and Higgins JP. 2011. Recommendations for examining and interpreting funnel plot asymmetry in meta-analyses of randomised controlled trials. BMJ 343(d4002. 10.1136/bmj.d4002

Stroup DF, Berlin JA, Morton SC, Olkin I, Williamson GD, Rennie D, Moher D, Becker BJ, Sipe TA, and Thacker SB. 2000. Meta-analysis of observational studies in epidemiology: a proposal for reporting. Meta-analysis Of Observational Studies in Epidemiology (MOOSE) group. JAMA 283(15):2008-2012.

Tewari A, Sooriakumaran P, Bloch DA, Seshadri-Kreaden U, Hebert AE, and Wiklund P. 2012. Positive surgical margin and perioperative complication rates of primary surgical treatments for prostate cancer: a systematic review and meta-analysis comparing retropubic, laparoscopic, and robotic prostatectomy. Eur Urol 62(1):115. 10.1016/j.eururo.2012.02.029

Thompson SG. 1994. Why sources of heterogeneity in meta-analysis should be investigated. BMJ 309(6965):13511355.

Tunis AS, Mclnnes MD, Hanna R, and Esmail K. 2013. Association of study quality with completeness of reporting: 
477

486

487

488

489

490

491

492

have completeness of reporting and quality of systematic reviews and meta-analyses in major radiology journals changed since publication of the PRISMA statement? Radiology 269(2):413-426. 10.1148/radiol.13130273

van Die MD, Bone KM, Williams SG, and Pirotta MV. 2014. Soy and soy isoflavones in prostate cancer: a systematic review and meta-analysis of randomized controlled trials. BJU Int 113(5b):E119-130. 10.1111/bju.12435

Zhang H, Han J, Zhu YB, Lau WY, Schwartz ME, Xie GQ, Dai SY, Shen YN, Wu MC, Shen F, and Yang T. 2016. Reporting and methodological qualities of published surgical meta-analyses. J Clin Epidemiol 70(4-16. 10.1016/j.jclinepi.2015.06.009

\section{Figure Legends}

Figure 1. Flow diagram of included meta-analyses

Figure 2. (a) Bar graph of percentage of meta-analyses that included each item from the PRISMA checklist. (b) Bar graph of percentage of meta-analyses that included each item from the AMSTAR tool. 


\section{Figure 1 (on next page)}

Figure 1. Flow diagram of included meta-analyses 
Records identified through database searching $(\mathrm{n}=641)$

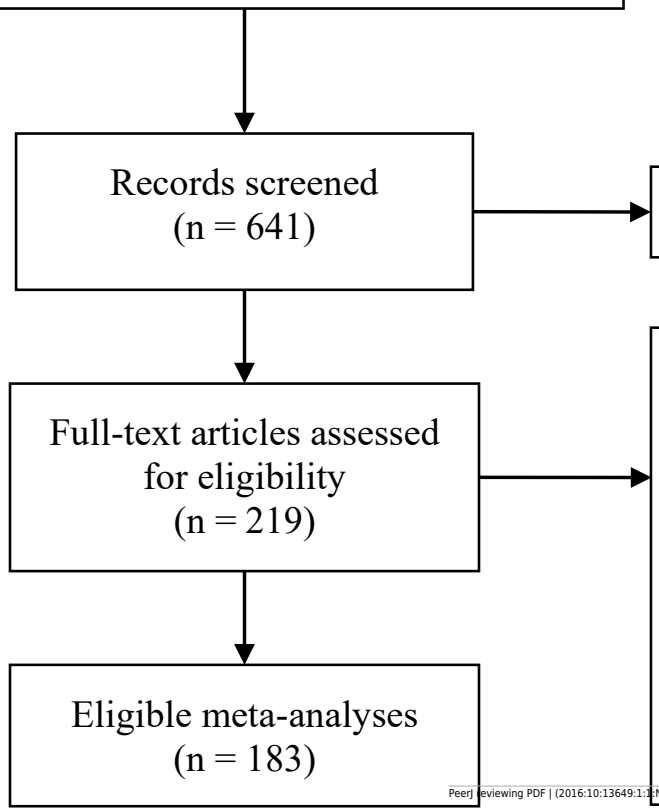

Records excluded on basis of titles and abstracts $(n=422)$

Full-text articles excluded, with reasons $(n=36)$ :

Systematic review without meta-analysis: 8

Original research: 2

Original research combined with meta-analysis: 3

Network meta-analysis: 5

Multiple group comparison meta-analysis: 2

Meta-analysis of single proportions: 8

Cochrane systematic review: 7

Animal study: 1 


\section{Figure 2 (on next page)}

Figure 2. (a) Bar graph of percentage of meta-analyses that included each item from the PRISMA checklist. (b) Bar graph of percentage of meta-analyses that included each item from the AMSTAR tool. 


\section{Table 1 (on next page)}

Table 1. Characteristics of included meta-analyses and quality scores assessed by the PRISMA checklist and AMSTAR tool 
1 Table 1. Characteristics of included meta-analyses and quality scores assessed by the PRISMA checklist

2 and AMSTAR tool

3

\begin{tabular}{|c|c|c|c|}
\hline Items & $\mathbf{N}(\%)$ & PRISMA, mean (SD) & $\begin{array}{l}\text { AMSTAR, mean } \\
\text { (SD) }\end{array}$ \\
\hline \multicolumn{4}{|l|}{ Journal } \\
\hline BJUI & $27(15 \%)$ & $22.85(2.13)$ & $7.93(1.14)$ \\
\hline EU & $44(24 \%)$ & $23.52(1.93)$ & $7.98(1.47)$ \\
\hline JEU & $21(11 \%)$ & $22.62(1.53)$ & $7.95(1.02)$ \\
\hline JPU & $2(1 \%)$ & $23(1.41)$ & $9(1.41)$ \\
\hline $\mathrm{JU}$ & $25(14 \%)$ & $22.52(2.37)$ & $7.2(1.68)$ \\
\hline NUUD & $3(2 \%)$ & $21.67(0.58)$ & $7.33(1.15)$ \\
\hline $\mathrm{U}$ & $25(14 \%)$ & $22.2(1.61)$ & $6.92(1.15)$ \\
\hline UL & $18(10 \%)$ & $22.67(2.22)$ & $7.44(1.24)$ \\
\hline UO & $6(3 \%)$ & $22.16(3.18)$ & $7.17(1.94)$ \\
\hline WJU & $12(7 \%)$ & $22.08(2.23)$ & $6.92(1.56)$ \\
\hline \multicolumn{4}{|l|}{ Origin region } \\
\hline Asia & $89(49 \%)$ & $22.82(1.94)$ & $7.56(1.28)$ \\
\hline Europe & $54(30 \%)$ & $22.78(2.05)$ & $7.80(1.42)$ \\
\hline North America & $29(16 \%)$ & $22.66(2.22)$ & $7.41(1.59)$ \\
\hline Oceania & $8(4 \%)$ & $22.13(2.9)$ & $7.13(2.10)$ \\
\hline South America & $3(2 \%)$ & $22.33(1.53)$ & $6.33(0.58)$ \\
\hline \multicolumn{4}{|l|}{ Origin country } \\
\hline Australia & $6(3 \%)$ & $22.33(3.01)$ & $7.17(2.14)$ \\
\hline Austria & $3(2 \%)$ & $22.67(3.06)$ & $7(1.73)$ \\
\hline Brazil & $4(2 \%)$ & $21.5(2.08)$ & $6(.82)$ \\
\hline Canada & $7(4 \%)$ & $22.29(1.98)$ & $7.57(1.51)$ \\
\hline China $\dagger$ & $82(45 \%)$ & $22.77(1.95)$ & $7.60(1.26)$ \\
\hline France & $3(2 \%)$ & $22.67(1.53)$ & $8(1)$ \\
\hline Italy & $17(9 \%)$ & $21.59(2.06)$ & $7.24(1.71)$ \\
\hline The Netherlands & $7(4 \%)$ & $24(1.73)$ & $7.86(0.69)$ \\
\hline UK & $20(11 \%)$ & $23.55(1.682)$ & $8.6(0.94)$ \\
\hline USA & $22(12 \%)$ & $22.77(2.33)$ & $7.36(1.65)$ \\
\hline Other Countries & $12(7 \%)$ & $23(1.65)$ & $7.08(1.51)$ \\
\hline \multicolumn{4}{|l|}{ Number of authors } \\
\hline$\geq 7$ & $93(51 \%)$ & $22.86(1.92)$ & $7.72(1.42)$ \\
\hline$<7$ & $90(49 \%)$ & $22.62(2.16)$ & $7.41(1.40)$ \\
\hline \multicolumn{4}{|l|}{ Statistician as coauthor } \\
\hline Yes (1) & $44(24 \%)$ & $22.68(2.45)$ & $7.48(1.64)$ \\
\hline No $(0)$ & $139(76 \%)$ & $22.76(1.91)$ & $7.60(1.34)$ \\
\hline \multicolumn{4}{|l|}{ Participating center } \\
\hline Single & $46(25 \%)$ & $22.57(1.76)$ & $7.48(1.21)$ \\
\hline Multiple & $137(75 \%)$ & $22.80(2.13)$ & $7.60(1.48)$ \\
\hline \multicolumn{4}{|l|}{ Subspecialty } \\
\hline Pediatric urology & $13(7 \%)$ & $21.77(2.59)$ & $7.15(2.03)$ \\
\hline Urologic oncology & $75(41 \%)$ & $23.12(2.16)$ & $7.65(1.50)$ \\
\hline Renal transplantation & $1(1 \%)$ & 22 & 7 \\
\hline Male infertility & $6(3 \%)$ & $20.5(1.05)$ & $6.33(1.03)$ \\
\hline
\end{tabular}




\begin{tabular}{|c|c|c|c|}
\hline Calculi & $39(21 \%)$ & $22.69(1.98)$ & $7.49(1.25)$ \\
\hline Female urology & $5(3 \%)$ & $23.4(1.14)$ & $8.8(1.09)$ \\
\hline Neurourology & $42(23 \%)$ & $22.67(1.72)$ & $7.67(1.16)$ \\
\hline Cannot classify & $2(1 \%)$ & $23(0)$ & $7.5(0.71)$ \\
\hline \multicolumn{4}{|l|}{ Funding support } \\
\hline Yes & $55(30 \%)$ & $23.31(2.04) *$ & $7.82(1.28)$ \\
\hline No & $128(70 \%)$ & $22.50(2.00)$ & $7.46(1.46)$ \\
\hline \multicolumn{4}{|l|}{ Number of included studies } \\
\hline$\geq 10$ & $101(55 \%)$ & $22.89(2.08)$ & $7.50(1.46)$ \\
\hline$<10$ & $82(45 \%)$ & $22.56(2.00)$ & $7.66(1.35)$ \\
\hline \multicolumn{4}{|l|}{ Included studies type } \\
\hline Only RCTs & $62(34 \%)$ & $22.82(1.92)$ & $7.95(1.27)$ \\
\hline RCTs \& non-RCTs & $50(27 \%)$ & $22.90(1.88)$ & $7.62(1.40)$ \\
\hline Only non-RCTs & $71(39 \%)$ & $22.56(2.27)$ & $7.20(1.46)$ \\
\hline \multicolumn{4}{|l|}{ Included studies type } \\
\hline Only RCTs & $62(34 \%)$ & $22.82(1.92)$ & $7.95(1.27) * *$ \\
\hline $\begin{array}{l}\text { RCTs \& non-RCTs or only } \\
\text { non-RCTs }\end{array}$ & $121(66 \%)$ & $22.70(2.11)$ & $7.37(1.44)$ \\
\hline \multicolumn{4}{|l|}{ Meta-analyses type } \\
\hline Interventional & $141(77 \%)$ & $22.60(1.96)$ & $7.64(1.37)$ \\
\hline Diagnostic & $12(7 \%)$ & $23.67(2.15)$ & $7.83(1.11)$ \\
\hline Incidence & $15(8 \%)$ & $23.40(2.20)$ & $7.07(1.71)$ \\
\hline Prognostic & $15(8 \%)$ & $22.73(2.46)$ & $7.2(1.61)$ \\
\hline \multicolumn{4}{|l|}{ Surgical intervention } \\
\hline Yes & $96(68 \% \vdots)$ & $22.46(1.86)$ & $7.56(1.36)$ \\
\hline No & $45(32 \% \ddagger)$ & $22.89(2.14)$ & $7.80(1.41)$ \\
\hline \multicolumn{4}{|l|}{ PRSIMA checklist } \\
\hline Yes & $2(1 \%)$ & $22.5(0.71)$ & $7.5(0.71)$ \\
\hline No & $181(99 \%)$ & $22.75(2.06)$ & $7.57(1.42)$ \\
\hline \multicolumn{4}{|l|}{ Followed PRSIMA } \\
\hline Yes & $55(30 \%)$ & $23.38(2.13) * *$ & $7.94(1.48) *$ \\
\hline No & $128(70 \%)$ & $22.47(1.95)$ & $7.41(1.35)$ \\
\hline \multicolumn{4}{|l|}{ Protocol and registration } \\
\hline Yes & $8(4 \%)$ & $26(1.19) * * *$ & $9.75(0.71) * * *$ \\
\hline No & $175(96 \%)$ & $22.59(1.95)$ & $7.47(1.36)$ \\
\hline \multicolumn{4}{|l|}{ "a priori" design } \\
\hline Yes & $22(12 \%)$ & $24.09(2.07) * * *$ & $9.14(1.13) * * *$ \\
\hline No & $161(88 \%)$ & $22.56(1.98)$ & $7.35(1.31)$ \\
\hline
\end{tabular}

$5 * \mathrm{P}<0.05 ; * * \mathrm{P}<0.01 ; * * * \mathrm{P}<0.001$

$6 \uparrow 81$ from mainland China, 1 from Taiwan,

$7 \$$ out of 141 


\section{Table 2 (on next page)}

Table 2. Univariate and multivariate logistic regression analyses of predictive factors associated with superior reporting quality (PRISMA) 
Table 2.Univariate and multivariate logistic regression analyses of predictive factors associated with superior reporting quality (PRISMA)

\begin{tabular}{|c|c|c|c|c|}
\hline \multirow[t]{2}{*}{ Variables } & \multicolumn{2}{|c|}{ Univariate } & \multicolumn{2}{|c|}{ Multivariate } \\
\hline & OR $(95 \% \mathrm{CI})$ & $P$ value & OR $(95 \% \mathrm{CI})$ & $P$ value \\
\hline Asia (ref. non-Asia) & $1.09(0.59,2.00)$ & 0.79 & & \\
\hline China (ref. non-China) & $1.02(0.55,1.89)$ & 0.95 & & \\
\hline No. of authors (continues) & $1.02(0.92,1.14)$ & 0.67 & & \\
\hline No. of authors $\geq 7$ (ref. $<7$ ) & $1.55(0.84,2.88)$ & 0.16 & & \\
\hline Presence of epi/stats professional (ref. absence) & $0.77(0.37,1.61)$ & 0.49 & & \\
\hline No. of participating center (continues) & $0.98(0.87,1.10)$ & 0.72 & & \\
\hline Multiple center (ref. single center) & $1.23(0.60,2.53)$ & 0.57 & & \\
\hline Urologic oncology (ref. other subspecialties) & $2.14(1.14,3.99)$ & 0.02 & $1.41(0.68,2.91)$ & 0.36 \\
\hline Funding support (ref. no) & $2.29(1.19,4.41)$ & 0.01 & $2.33(1.16,4.69)$ & 0.02 \\
\hline No. of included studies (continues) & $1.00(0.99,1.01)$ & 0.91 & & \\
\hline No. of included studies $\geq 10$ (ref. $<10$ ) & $1.31(0.71,2.45)$ & 0.38 & & \\
\hline $\begin{array}{l}\text { Included only RCTs } \\
\text { (ref. RCTs \& non-RCTs or only non-RCTs) }\end{array}$ & $1.00(0.52,1.91)$ & 1.00 & & \\
\hline Interventional (ref. non-interventional) & $0.47(0.23,0.94)$ & $\mathbf{0 . 0 3}$ & $0.45(0.19,1.03)$ & 0.06 \\
\hline Surgical intervention (ref. non-surgical intervention) & $0.58(0.27,1.23)$ & 0.16 & & \\
\hline Followed PRSIMA (ref. no) & $2.04(1.07,3.94)$ & $\mathbf{0 . 0 3}$ & $1.88(0.92,3.87)$ & 0.09 \\
\hline "a priori" design (ref. no) & $3.30(1.32,8.23)$ & 0.01 & $3.74(1.40,10.02)$ & 0.01 \\
\hline
\end{tabular}




\section{Table 3(on next page)}

Table 3. Univariate and multivariate logistic regression analyses of predictive factors associated with superior methodological quality (AMSTAR) 
Table 3.Univariate and multivariate logistic regression analyses of predictive factors associated with superior methodological quality (AMSTAR)

\begin{tabular}{|c|c|c|c|c|}
\hline \multirow[t]{2}{*}{ Variables } & \multicolumn{2}{|c|}{ Univariate } & \multicolumn{2}{|c|}{ Multivariate } \\
\hline & OR $(95 \% \mathrm{CI})$ & $\mathrm{P}$ value & OR $(95 \% \mathrm{CI})$ & P value \\
\hline Asia (ref. non-Asia) & $0.77(0.40,1.49)$ & 0.43 & & \\
\hline China (ref. non-China) & $0.75(0.38,1.46)$ & 0.40 & & \\
\hline No. of authors (continues) & $1.15(1.02,1.29)$ & 0.02 & $1.00(0.87,1.16)$ & 0.95 \\
\hline No. of authors $\geq 7$ (ref. $<7$ ) & $1.69(0.87,3.31)$ & 0.12 & & \\
\hline Presence of epi/stats professional (ref. absence) & $1.25(0.59,2.64)$ & 0.57 & & \\
\hline No. of participating center (continues) & $1.11(1.00,1.25)$ & 0.06 & & \\
\hline Multiple center (ref. single center) & $1.63(0.72,3.71)$ & 0.24 & & \\
\hline Urologic oncology (ref. other subspecialties) & $2.07(1.06,4.04)$ & $\mathbf{0 . 0 3}$ & $2.05(0.92,4.58)$ & 0.08 \\
\hline Funding support (ref. no) & $1.59(0.79,3.19)$ & 0.19 & & \\
\hline No. of included studies (continues) & $1.00(0.99,1.01)$ & 0.85 & & \\
\hline No. of included studies $\geq 10$ (ref. $<10$ ) & $1.06(0.55,2.06)$ & 0.86 & & \\
\hline $\begin{array}{l}\text { Included only RCTs } \\
\text { (ref. RCTs \& non-RCTs or only non-RCTs) }\end{array}$ & $1.78(0.91,3.51)$ & 0.10 & & \\
\hline Interventional (ref. non-interventional) & $1.00(0.46,2.19)$ & 1.00 & & \\
\hline Surgical intervention (ref. non-surgical intervention) & $0.82(0.37,1.81)$ & 0.63 & & \\
\hline Followed PRSIMA (ref. no) & $3.80(1.90,7.64)$ & $<0.001$ & $2.94(1.33,6.47)$ & 0.01 \\
\hline "a priori” design (ref. no) & $19.65(6.19,62.29)$ & $<0.001$ & $17.37(4.98,60.56)$ & $<0.001$ \\
\hline
\end{tabular}

